\title{
POTENCIALIDAD DE PSEUDOSUCCINEA COLUMELLA (SAY, 1817) (MOLLUSCA: GASTROPODA: LYMNAEIDAE) EN LA TRANSMISIÓN DE LA FASCIOLIASIS HUMANA EN REPÚBLICA DOMINICANA
}

\section{Potentiality of Pseudosuccinea columella (Say, 1817) (Mollusca: Gastropoda: Lymnaeidae) in the transmission of human fascioliasis in the Dominican Republic}

\section{Mercedes de Vargas Castro ${ }^{1 *}$, Jairo Martínez La Hoz ${ }^{2 * *}$, Jean Castillo ${ }^{1 * * *}$, Dolores Paulino ${ }^{2 * * * *}$, Pedro María Alarcón-Elbal ${ }^{3 n}$, Antonio A. Vázquez ${ }^{44}$}

Recibido: 12 enero, 2019 • Aprobado: 26 febrero, 2019

Cómo citar: Vargas Castro M de, Martínez La Hoz J, Castillo J, Paulino D, Alarcón-Elbal PM, Vázquez AA. Potencialidad de Pseudosuccinea columella (Say, 1817) (Mollusca: Gastropoda: Lymnaeidae) en la transmisión de la fascioliasis humana en República Dominicana. cysa [Internet]. 25 de julio de 2019 [citado 26 de julio de 2019];3(2): 9-16.

Disponible en: https://revistas.intec.edu.do/index.php/cisa/article/view/1474

\section{Resumen}

Introducción: en el paisaje epidemiológico de la fascioliasis humana, dos aspectos son de fundamental importancia: a) presencia de hospederos definitivos (especialmente ganado bovino y humanos) parasitados con adultos del tremátodo $\mathrm{y}$; b) presencia de hospederos intermediarios invertebrados, moluscos de la familia Lymnaeidae, en los que se desarrollan los estadios larvarios, además de plantas acuáticas de consumo humano.

Material y métodos: con el objetivo de prevenir brotes de fascioliasis humana mediante la correcta identificación de aspectos de interés epidemiológico, un humedal fue seleccionado en el sector de Palo Blanco, municipio de Jarabacoa, provincia de La Vega, en el cual se realizó un estudio malacológico en octubre de 2016, en busca de especies de moluscos limneidos e hidrófitos asociados. En el laboratorio se obtuvieron los índices de infección natural y experimental a Fasciola hepatica.

Resultados: se identificó el limneido Pseudosuccinea columella, en proporciones de 250 especímenes por persona/hora.

1. Instituto Tecnológico de Santo Domingo, Laboratorio de Fasciola hepatica. INTEC 2. Universidad Autónoma de Santo Domingo, Facultad de Ciencias Agrícolas y Veterinarias, UASD

3. Instituto de Medicina Tropical \& Salud Global (IMTSAG), Universidad Iberoamericana (UNIBE), Santo Domingo, República Dominicana

4-Instituto de Medicina Tropical "Pedro Kouri”", IPK. La Habana, Cuba

\begin{abstract}
Introduction: in the epidemiological landscape of human fascioliasis, two aspects are of fundamental importance: a) presence of definite hosts (especially cattle and humans) parasitized with trematode adults and b) presence of invertebrate intermediate hosts, mollusks of the family Lymnaeidae, in which larval stages develop, in addition to aquatic plants for human consumption.
\end{abstract}

Material and methods: in order to prevent outbreaks of human fascioliasis by correctly identifying aspects of epidemiological interest, a wetland was selected in the sector of Palo Blanco, municipality of Jarabacoa, province of La Vega, in which a malacological study was carried out in October 2016, in search of limneid mollusk species and associated hydrophytes. Natural and experimental infection rates to Fasciola hepatica were obtained in the laboratory.

Results: the lymnaeid Pseudosuccinea columella was identified, in proportions of 250 specimens collected per

Correo-e de los autores:

* Autor para correspondencia: mercedesvargasc24@gmail.com

** jairillo88@gmail.com

*** jccastillo.u@gmail.com

**** dolores100093991@gmail.com

$\Omega$ pedro.alarcon@uv.es

$\Delta$ applesnail@hotmail.fr 
El vegetal predominante fue el berro (Nasturtiun officinale), cultivado comercialmente en tres cuadrantes del humedal y en el otro cuadrante se observaron bovinos pastando. El índice de infección experimental de los moluscos fue de $94 \%$ a la cepa simpátrica de F. hepatica de Dajabón; no se encontraron moluscos parasitados naturalmente.

Conclusiones: Pseudosuccinea columella debe considerarse como un molusco de relevancia epidemiológica en la transmisión de la fascioliasis en República Dominicana, a tenor de su dispersión y elevado índice de infección experimental obtenido en el presente estudio.

Palabras clave: Fasciola hepática; molusco limneido; zoonosis; berro; Antillas Mayores.

\section{Introducción}

La fascioliasis o distomatosis hepática es una trematodiasis zoonótica, desatendida, producida por la duela del hígado Fasciola hepatica (Linnaeus, 1758) (Trematoda: Digenea); popularmente conocida por los ganaderos dominicanos como «cucaracha de hígado». Esta parasitosis emergente y reemergente, deteriora considerablemente la salud de rumiantes y humanos en todo el mundo tropical y subtropical ${ }^{1}$, con un incremento constante de las prevalencias e incidencias humanas en todos los continentes ${ }^{2}$ y una patogenicidad mayor de lo hasta ahora conocida ${ }^{3}$. Es adquirida por la ingestión de plantas acuáticas, principalmente por el consumo de berros (Nasturtium officinale) y lechuga (Lactuca sativa), en ensaladas crudas, y por ingestión de jugos de alfalfa (Medicago sativa) contaminados con metacercarias, que constituye la fase infectante de F. hepatica. Después de la ingestión de las metacercarias y su posterior desenquistamiento en el duodeno, estas larvas atraviesan la pared del intestino delgado y migran por el peritoneo del humano hasta alcanzar el hígado; atraviesan la cápsula de Glisson, la cual perforan, y se refugian en los canalículos biliares, donde se desarrollan a adultos. En su recorrido producen focos necróticos y fibrosos relacionados con la migración de las larvas, presentándose necrosis debido al traumatismo provocado por la acción física de los trematodos. person/hour. The predominant vegetable was the watercress (Nasturtiun officinale), commercially cultivated in three quadrants of the wetland and in the other quadrant were observed cattle grazing. The rate of experimental infection of mollusks was $94 \%$ to the sympatric strain of $F$. hepatica of Dajabón; no naturally parasitized mollusks were found.

Conclusions: Pseudosuccinea columella should be considered as a mollusk of epidemiological relevance in the transmission of fascioliasis in the Dominican Republic, due to its dispersion and high rate of experimental infection obtained in the present study.

Keywords: Fasciola hepatica; hepatic trematodiasis; lymnaeid mollusks; zoonosis; watercress, Greater Antilles.

Según Kleinman, Petriokowsky y Prepelit ${ }^{7}$, en los países donde esta enfermedad es endémica se produce un grave perjuicio económico, entre otros, debido al decomiso de hígados no aptos para el consumo humano, ya que se encuentran endurecidos por fibrosis, con punteados purulentos y, en consecuencia, son desagradables a la vista.

En República Dominicana la situación en humanos es desconocida, aunque se presentan casos esporádicos que acuden a la consulta. De Vargas Castro (inédito) ha señalado la enfermedad en humanos en la región central de la isla, concretamente en Constanza, provincia de La Vega, en seis individuos pertenecientes a una misma familia, los cuales consumieron la misma ensalada de berros (epidemia familiar), además de Santiago de los Caballeros y otro caso en el municipio de Cotuí, provincia de Sánchez Ramírez. Estos casos evidencian que existe un subregistro de la enfermedad humana en el país.

Teniendo en cuenta la importancia sanitaria de los moluscos dulceacuícolas y la escasez de investigaciones científicas en relación con estos publicadas en el país, el presente estudio tuvo como objetivo determinar la malacofauna existente en una zona de montaña de El Cibao, concretamente en Jarabacoa, y determinar la potencialidad de las especies clave en la transmisión de la fascioliasis humana en República Dominicana. 


\section{Materiales}

\section{Área de estudio}

El área seleccionada para el estudio correspondió al valle intramontano de Jarabacoa, provincia de La Vega, con un área de $23 \mathrm{~km}^{2}$ y $330 \mathrm{~m}$ de altitud, en el nordeste de la Cordillera Central. Suelo de origen lacustre y aluviales, clima de bosque húmedo tropical y temperatura media anual de $21.8^{\circ} \mathrm{C}$. Se seleccionó un humedal antrópico de 3,42 ha, ubicado en el sector de Palo Blanco (19 06'23.55"N $70^{\circ} 37^{\prime} 42.86$ "W, $586 \mathrm{msnm}$ ) y constituido por cuatro predios: tres dedicados al cultivo de berros y otro al pastoreo de ganado, principalmente bovinos. Los predios están limitados por los bordillos de las aceras y divididos por subcuadrantes de montículos de tierra con centros cenagosos de $30 \times 15 \mathrm{~m}$. El agua surge de un manantial con pendiente suave, limpia y ondulada, que corre atravesando cada cuadrante a manera de vasos comunicantes. El berro se cultiva de forma bien planificada, con cortes periódicos después de su floración.

\section{Recolección de moluscos}

Durante octubre de 2016 se procedió al registro de moluscos y especies de vegetales creciendo dentro del hábitat. Se siguió la metodología estándar según Malek ${ }^{9}$, análisis conquiológicos y conteos de moluscos persona/hora. De igual manera, se registró la presencia de otras especies de interés médico biológico, tanto acuáticas como terrestres. Se usaron pinzas de presión suave, para no maltratar los moluscos. Las muestras fueron conservadas en papel toalla húmedo y colocadas en envases plásticos, para ser llevadas sin deterioro hasta el laboratorio.

\section{Infección experimental en condiciones de laboratorio}

Los moluscos limneidos fueron divididos en tres lotes: a) unos fijados en alcohol etílico al $80 \%$, para el Museo de Moluscos de INTEC; b) otro grupo de 30 moluscos fue colocado en cajas de Petri con algas cultivadas en el laboratorio (cedidas por el IPK de
La Habana, Cuba) para obtener la F1 sin contaminación natural y, posteriormente, determinar el índice de infección experimental; y c) otro lote fue disecado para identificar microscópicamente posibles infecciones naturales de helmintos a través de la visualización de redias y/o cercarias.

Treinta y seis (36) moluscos pertenecientes a la especie Pseudosuccinea columella (Say, 1817) con 7-10 días de nacidos, alimentados con lechuga dos veces por semana y algas cultivadas en el laboratorio, a temperatura estable de $24^{\circ} \mathrm{C}$ y luz blanca permanentes, fueron expuestos a la infección en masa de miracidios de F. hepatica del linaje de Dajabón, República Dominicana. Estos miracidios fueron obtenidos a partir de un aislado bovino, concretamente de huevos en la bilis de hígados de vacas infectadas, lavados varias veces con solución salina $0.85 \%$ y guardados en refrigeración hasta 15 días antes de la planificación de la infección. Después, fueron colocados a temperatura ambiente para el desarrollo de las larvas. Estos huevos fueron expuestos a la luz blanca de una lámpara para estimular la salida de los miracidios. Estas larvas fueron obtenidas con pipetas especiales, y colocadas en placas de Petri pequeñas junto a los moluscos por un período de dos horas. Posteriormente se colocaron en placas de Petri y se alimentaron de la misma manera que fue descrita arriba. Pasados 15 días de la infección experimental fueron disecados y estudiados bajo el microscopio a 40x, donde la presencia o ausencia de redias fue notada. Los moluscos se observaron diariamente con el fin de determinar el índice de mortalidad.

\section{Resultados}

\section{Caracterización de la malacofauna dulceacuicola}

De los moluscos capturados, fue preponderante el limneido P. columella (Figura 1), en elevada proporción, de 250 persona/hora; se mostraban sobre el fondo lodoso y adheridos a los tallos de las plantas de berros (Figura 2). Otras especies de moluscos 
estuvieron representadas: de la familia Thiaridae se recogieron conchas vacías de Tarebia granifera (Lamarck, 1816) y Melanoides tuberculata (Muller, 1774), además de conchas de Marisa cornuarietis (Linnaeus, 1758) y el Ampullariidae Pomacea canaliculata (Pilsbry, 1927), este último en plena oviposición. Especies terrestres fueron apreciadas sobre los montículos de tierra por encima del nivel del agua: Subulina octona (Bruguière 1798), Bulimulus sp. y Succinea sp.

\section{Infección experimental en condiciones de laboratorio}

El índice de infección experimental de los moluscos fue de $94 \%$ (34/36) a la cepa simpátrica de F. hepatica de Dajabón. No se encontraron moluscos parasitados naturalmente.

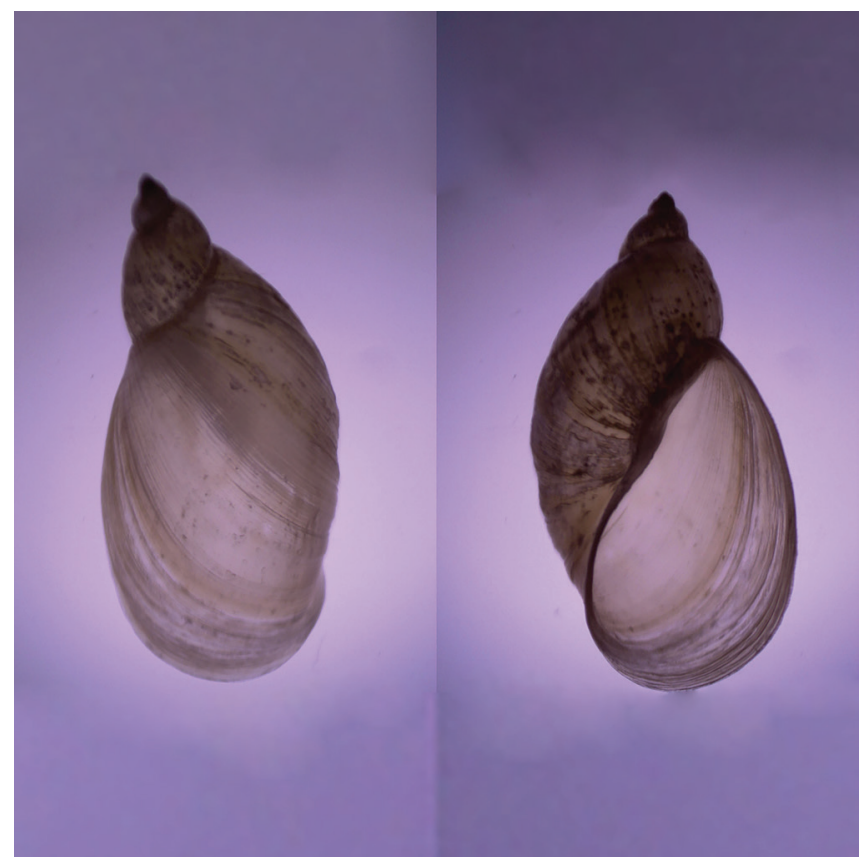

Figura 1. Concha de Pseudosuccinea columella.

Fuente: elaboración propia.

Único limneido identificado en el área de muestreo. Nótese la última vuelta del cuerpo que ocupa 2/3 partes del total de la concha. Estos moluscos exhiben una amplia capacidad de colonización de otros nichos ecológicos presentes en la comunidad.

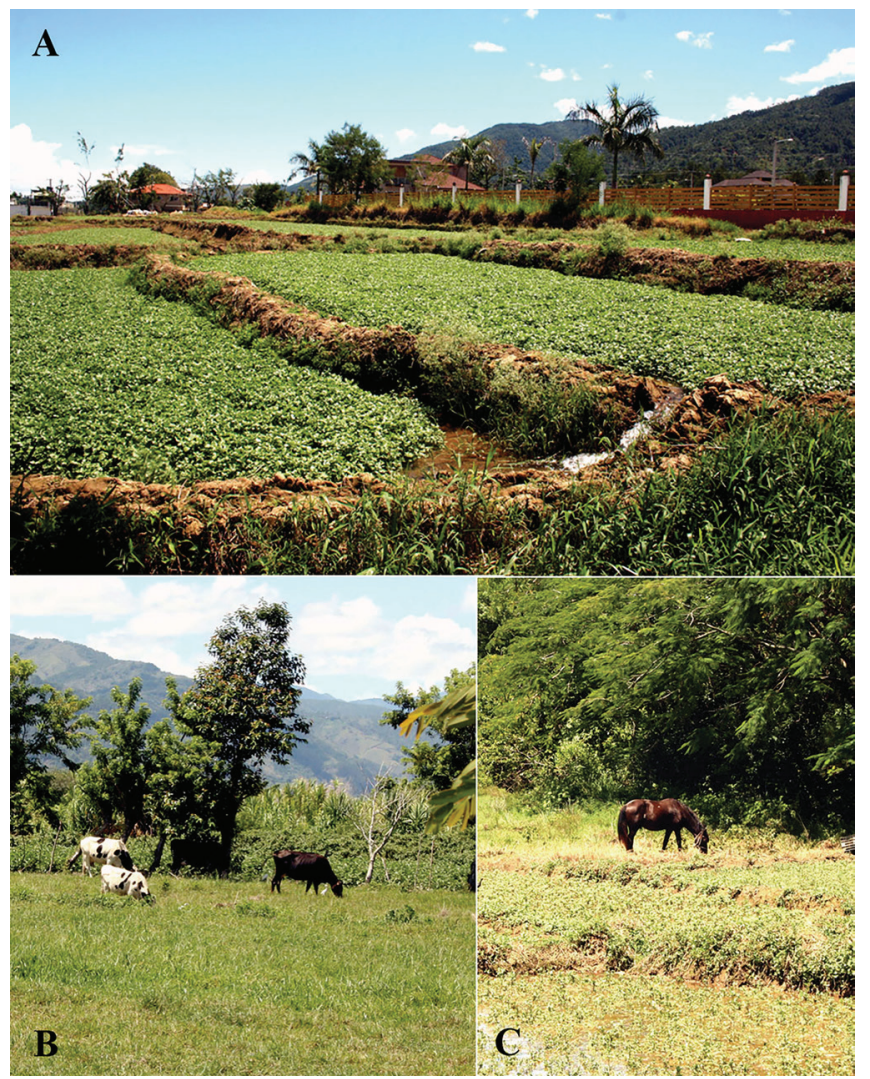

Figura 2. Área de muestreo, donde: A) Detalle de los cultivos de berros en la etapa de floración, listo para el corte; B) Ganado vacuno pastando en un espacio contiguo al cultivo de berros, y C) Ganado caballar pastando dentro de cultivos incipientes de berros.

Fuente: elaboración propia.

\section{Discusión}

El reconocimiento de las especies de moluscos transmisores de la fascioliasis es de fundamental importancia para prevenir y controlar cualquier brote epidémico de la enfermedad. Los limneidos vectores continúan representando un factor crítico en su transmisión, e inclusive, generando confusión en la taxonomía ${ }^{10}$, lo cual ha sido evidenciado por el uso de técnicas moleculares en la identificación de las especies.

En un país pueden existir varias especies de limneidos, siendo tan solo una la responsable de la transmisión del parásito ${ }^{11,14}$; en otros, una o dos 
especies podrían mantener la endemia ${ }^{15}$, y otras especies son completamente refractarias al desarrollo de miracidios de F. hepatica, como es el caso de Galba schirazensis (Küster, 1862) en República Dominicana (De Vargas Castro, inédito), y en España e Irán, según Bargues et. al. ${ }^{10}$, mientras que esa misma especie en Ecuador ${ }^{16}$ se presenta sensible al parásito en condiciones naturales, transmitiendo la enfermedad y sugiriendo una adaptación natural del parásito al molusco ${ }^{16}$. Existe cierta discusión entre los investigadores al respecto; solo las técnicas moleculares son capaces de identificar una especie con exactitud. Las técnicas de identificación meramente conquiológicas han pasado a un segundo plano en estos $\operatorname{casos}^{17}$.

En República Dominicana y otras islas de las Antillas Mayores, la especie tradicionalmente involucrada en la transmisión ha sido Galba cubensis (Pfeiffer, 1839) (anteriormente Lymnaea cubensis) ${ }^{14}$. Sin embargo, se desconoce el papel desempeñado por P. columella en condiciones naturales. Este limneido fue reportado por primera vez en República Dominicana en 1986 por Gómez, De Vargas y Malek ${ }^{18-20}$, y seńalado como un posible hospedero intermediario. Por entonces, la especie tan solo fue observada en el sur del país: en el Jardín Botánico de Santo Domingo y en el sector La Laguna, en Quita Sueño de Haina, San Cristóbal; con un índice poblacional muy bajo y negatividad al parásito, sugestivo de introducción reciente, posiblemente con la importación de plantas ornamentales de agua dulce desde EE. UU., su localidad tipo. En ambas localidades, por entonces, no existía riesgo de infección por la ausencia de otro factor indispensable en la transmisión: bovinos y otros vertebrados aportando huevos del parásito.

En la actualidad, esta especie de molusco se ha expandido a otras áreas del país; se ha encontrado en 15 hábitats diferentes (De Vargas Castro et al., en preparación) y distantes del criadero original, demostrando su capacidad invasora señalada; además, desde países e islas tan lejanas como Australia,
Nueva Zelanda, Francia ${ }^{21}$ y otros lugares a nivel mundial. En el continente americano se encuentra distribuida en varios países de Sudamérica: Brasil ${ }^{22}$, Venezuela ${ }^{23}$, Colombia ${ }^{24}$, Argentina ${ }^{25}$ y Perú ${ }^{26}$. Varios autores han señalado su presencia en Chile, Costa Rica, México ${ }^{5}$, Uruguay ${ }^{27}$. En Las Antillas Mayores: Cuba ${ }^{4,15}$, Puerto Rico ${ }^{11,12}$, República Dominicana $^{13,14,18,20}$ y Jamaica. En el Arco Antillano en las Islas Vírgenes ${ }^{28}$, además de lugares tan lejanos como: Australia, África y Europa. En Francia, Pointier, Coustau, Rondelaud y Theron ${ }^{21}$ la reportan en el Jardín Botánico y posteriormente la capturan en el río ${ }^{18}$; estos especímenes fueron criados en el laboratorio y su progenie presentó $100 \%$ de susceptibilidad a la cepa francesa de $F$. hepatica y $0 \%$ de mortalidad en condiciones experimentales. En nuestra investigación obtuvimos $94 \%$ (34/36) de susceptibilidad y $0 \%$ de mortalidad ante el linaje de F. hepatica de Dajabón.

Aunque el limneido clásicamente responsabilizado por transmitir la fascioliasis en República Dominicana es $G$. cubensis $^{14}$, no debe subestimarse la participación de $P$. columella en la trasmisión. Esta especie podría contribuir a la expansión de la enfermedad, como ha sucedido en otros países, especialmente los sudamericanos ${ }^{22,23,25}$.

Las actividades humanas son los principales factores responsables por la invasión de medios ambientes acuáticos por moluscos de agua dulce ${ }^{18,21}$. Es un fenómeno que ha aumentado considerablemente en los últimos años a nivel mundial con el comercio de peces, plantas ornamentales y el cultivo de berros, con trasplantes de un vivero a otro. Ejemplo de ello, hoy día, en República Dominicana, ha habido una invasión de ecosistemas de agua dulce por la especie exótica invasora Anodonta grandis (Say, 1829) ${ }^{18}$, molusco bivalvo, comestible, introducido al país con tilapias traídas desde Egipto por la Secretaría de Agricultura; además, otro ejemplo clásico es la invasión de Galba truncatula (Müller, 1774) traída desde Europa con el comercio de reses europeas hacia Sudamérica. 
En humedales de la región Central Andina de Colombia, Giraldo-Pinzón y Álvarez-Mejía ${ }^{24}$ registraron que el berro es la planta más asociada al limneido $P$. columella, en cuerpos de agua dulce de corrientes limpias con elevado crecimiento predominante de esta especie de planta silvestre. Nuestros resultados coinciden con los registros de otros países, además de que esta planta puede hospedar moluscos de otras especies. A nivel mundial, el berro es la planta acuática más relacionada con $P$. columella, más que con ninguna otra especie de limneido ${ }^{24}$. En nuestro país el berro es parte de la dieta de los dominicanos y al consumirse fresco se potencializa como factor dispersante de la fascioliasis humana, no solo en el área de estudio, sino en todo el país. Una posible acción para evitar posibles infecciones sería no consumir berros u otras plantas acuáticas silvestres mal lavadas, siendo lo más aconsejable realizar una adecuada cocción de los mismos. No obstante, también se ha demostrado la presencia de metacercarias flotantes en agua ${ }^{29}$, por lo que el consumo de agua sin hervir pudiera ser otro factor de riesgo para adquirir esta infección.

Un aspecto importante a resaltar en nuestra investigación, es la presencia de ganado pastando a escasos metros de los criaderos de moluscos y cultivos de berros (Figura 2), lo cual aumenta la probabilidad de transmisión de fascioliasis en la población humana.

\section{Conclusiones}

A pesar del papel protagónico de G. cubensis en la transmisión de la fascioliasis en República Dominicana, $P$. columella debe considerarse como un molusco de relevancia epidemiológica, a tenor de su dispersión en el territorio dominicano y el elevado índice de infección experimental obtenido en el presente estudio.

En Jarabacoa existen condiciones ecológicas, biológicas y culturales para la aparición de una epidemia de fascioliasis humana, con posibilidad de expansión a otras poblaciones mediante el comercio de berros, ya que coinciden en un mismo contexto hospederos definitivos e intermediarios, y la consabida planta cultivada para consumo humano.

Teniendo en cuenta la carencia de información sobre la fascioliasis humana en el país, es menester seguir promoviendo la realización de estudios parasitológicos y malacológicos, gracias a los cuales pueda arrojarse luz sobre la situación actual de esta zoonosis.

\section{Agradecimientos}

El presente trabajo se deriva del proyecto «Transmisión de Fasciola hepatica (Trematoda: Digenea), por sus moluscos hospederos intermediarios, (Gastropoda: Lymnaeidae), en el Caribe Insulan, Fondo Nacional de Innovación y Desarrollo Científico y Tecnológico (FONDOCyT) del Ministerio de Educación Superior, Ciencia y Tecnología (MESCyT). Agradecemos la colaboración de todos los estudiantes que han participado en el proyecto, así como a las contribuciones de INTEC, UASD, UNIBE e IPK.

\section{Bibliografía}

1. Carrada Bravo T. Fasciola hepatica: Ciclo biológico y potencial biótico. Revista Mexicana de Patología Clínica. 2007;54(1): 21-7.

2. Esteban JG, Flores A, Angles R, Mas-Coma S. High endemicity of human Fasciolosis between Lake Titicaca and La Paz valley, Bolivia. Transactions of The Royal Society of Tropical Medicine and Hygiene. 1999;93(2): 151-6.

3. González LC, Esteban JG, Bargues MD, Ortiz P, Náquira C, Mas-Coma S. Hyperendemic human fascioliasis in Andean Valley: an altitudinal transect analysis in children of Cajamarca Province, Peru. Acta Tropica. 2011;120(1-2): 119-29.

4. Díaz-Fernández R, González-Rubio D, MillánAlvarez LM, Garcés-Martínez M, Medina-Aguilar RL, Millán JC. Íctero obstructivo, Fasciola hepatica: presentación de un caso. Revista Cubana de Medicina Tropical. 2005;57(2): 151-3. 
5. Carrada-Bravo T. Fascioliasis: Diagnóstico, epidemiología y tratamientos. Revista de Gastroenterología Mexicana. 2003;68(2): 135-42.

6. Mas-Coma S, Esteban JG, Bargués MD. Epidemiología de la fascioliasis humana: Revisión y propuesta de nueva clasificación. Bulletin of the World Health Organization. 1999;77(4): 340-6.

7. Kleinman F, Petriokowsky S, Prepelit Chi L. Dinamics of Fasciola hepatica transmission in the Andean Patagonian Valleys, Argentina. 2006. Veterinary Parasitology, 2006;145(3-4): 274-86.

8. Marcos-Raymundo LA, Marco-Flores V, Terashima A, Samalvides F, Miranda E, Tantalean M, et. al. Hiperendemicidad de Fasciolosis humana en el Valle de Mantaro, Perú: factores de riesgo de la infección por Fasciola hepática. Revista de Gastroenterología del Perú. 2004;24(2): 6-20.

9. Malek EA. Laboratory guide and notes for medical malacology. Minneapolis: Burgess Publishing Co.; 1962.

10. Bargues MD, Artigas P, Khoubbane M, Flores R, Gloer P, Rojas-Garcia R, Ashrafi K, Falkner G, Mas-Coma S. Lymnaea schirazensis, an overlooked snail distorting fascioliasis data: genotype, phenotipe, ecology, worldwide spread, susceptibility, applicability. PLoS One. 2011;6(9): e24567.

11. Hillyer GV. Fascioliasis in Puerto Rico. [A review.] 1981. Boletín de la Asociación Médica de Puerto Rico. 1981;73: 94-101

12. León-Dancel D. Life history of Lymnaea columella (Say) and its experimental infection with Fasciola hepatica (L.). Journal of Agriculture of University of Puerto Rico. 1970;54(2): 297-305.

13. Ueno H, Alvarez-V JM, Mergen AMR, Sánchez VM. Observation on the prevalence of parasitic diseases in cattle especially fascioliasis, in the Dominican Republic. National Institute of Animal Health Quarterly. 1973;13(2): 59-68.

14. Martínez MA, Artigas P, Mateo L, Chagas V, Adam S, Mas-Coma S , Bargues MD. Identificación de Lymnaea cubensis en zonas endémicas de Fascioliasis en República Dominicana. XIX Congreso de la Sociedad Española de Parasitología; 23, 24 y 25 de julio de 2015; Vitoria-Gasteiz (España). SOCEPA; 2015.

15. Vásquez-Capote R, Diéguez Fernández L, DelRisco-Barrios U, Fimia-Duarte R, Vásquez-Perera AA. Pseudosuccinea columella (Say 1817) (Mollusca: Gastropoda: Lymnaeidae) en Camagüey. Veterinaria y Zootecnia. 2013;7(2): 63-74.

16. Caron Y, Celi-Erazo M, Hurtrez-Boussès S, Lounnas M, Pointier J, Saegerman C, Losson B, Benítez-Ortíz W. Is Galba schirazensis (Mollusca, Gastropoda) an intermediate host of Fasciola hepatica (Trematoda, Digenea) in Ecuador? Parasite. 2017;24: 24.

17. Bargues MD, González LC, Artigas P, Mas-Coma S. A new baseline for fascioliasis in Venezuela: lymnaeid vectors ascertained by DNA sequencing and analysis of their relationships with human and animal infection. Parasites \& Vectors. 2011;4: 200.

18. Gómez JD, De-Vargas M, Malek EA. Moluscos de agua dulce de República Dominicana. Colección Ambiente y Sociedad No 2. Santo Domingo: Universidad Autónoma de Santo Domingo; 1986.

19. Gómez JD, De-Vargas M, Malek EA. Pseudosuccinea columella new record Lymnaeidae in the Dominican Republic and transmission of fascioliasis in the Caribbean region. Nautilus. 1986;100(2): 66-8.

20. GómezJD, De-Vargas M, MalekEA. Freshwater mollusks of the Dominican Republic. Nautilus. 1986;100(4): 130-4. 
21. Pointier JP, Coustau C, Rondelaud D, Theron A. Pseudosuccinea columella (Say 1817) (Gastropoda Lymnaeidae), snail host of Fasciola hepatica: First record for France in the Wild. Parasitology Research. 2017;101(5): 1389-92.

22. Ueta MT. Ocorrência de infecção natural de Fasciola hepatica Linnaeus, 1758 em Lymnaea columella Say, 1817 no Vale do Paraíba, SP, Brasil. 1980. Revista Saude Pública. 1980;14(2): 230-3.

23. Guta J, Pérez A, Lecuna J, García M, Aguirre A, Armas S. Prevalencia de Fasciola hepática en ganadería de altura en Bailadores, Mérida, Venezuela. Revista Electrónica Veterinaria. 2011;12(12): 1-8.

24. Giraldo-Pinzón E, Álvarez-Mejía L. Registro de plantas hospederas de caracoles Lymnaeidae (Mollusca: Gastropoda) vectoras de Fasciola hepática (Limnaeus,1758) en humedales de la Región Central andina colombiana. Veterinaria y Zootecnia. 2013;7(2): 63-74.

25. Moriena RA, Álvarez JD, Pietrokousky D, Rubel D, Prepelitchi L, Racioppi OM, Winiversky C. Presencia de Pseudosuccinea columella naturalmente infestada con Fasciola hepatica en Santo Tomé (Corrientes, Argentina) 2008 Revista Veterinaria. 2008;19(2): 147-9.
26. Espinosa JR, Terashima A, Herrera-Velit P, Marcos LA. Fasciolosis humana y animal en Perú, impacto en la economía de las zonas endémicas. Revista Peruana de Medicina Experimental y Salud Pública. 2010;27(4): 605-12.

27. Armúa-Fernández MT, Castro $\mathrm{O}$, Correa $\mathrm{O}$, Carvalho L, Mangold A, Sanchís J, Venzal JM. First molecular characterization of Galba neotropica in Uruguay. Revista FAVE - Sección Ciencias Veterinarias. 2016;15: 9-13.

28. Vázquez AA, Sánchez-Noda J. Manual de Malacología Médica. La Habana: Instituto de Medicina Tropical "Pedro Kourî"; 2010.

29. Rojas L, Vázquez A, Domenech I, Robertson LJ. Fascioliasis: can Cuba conquer this emerging parasitosis? Trends in Parasitology. 2009;26(1): 26-34. 